\title{
Multi-adjoint concept lattices
}

\author{
Jesús Medina \\ Dept. Mathematics, University of Cádiz, Spain* \\ jesus.medina@uca.es
}

\begin{abstract}
Multi-adjoint concept lattices were introduced [2,3] as a new general approach to formal concept analysis, in which the philosophy of the multi-adjoint paradigm $[1,4]$ to formal concept analysis is applied. With the idea of providing a general framework in which different approaches could be conveniently accommodated, the authors worked in a general non-commutative environment; and this naturally lead to the consideration of adjoint triples, also called implication triples or bi-residuated structure as the main building blocks of a multi-adjoint concept lattice.

In recent years there has been an increased interest in studying formal concept analysis on the perspective of using non-commutative conjunctors. This is not a mere mathematical generalization, but a real need since, for instance, when one learns a conjunction from examples it is not unusual that the resulting conjunction does not satisfy commutativity. Different authors have argued in favour of considering non-commutative conjunctors. Actually, there exist quite reasonable examples of non-commutative and even non-associative conjunctors defined on a regular partition of the unit interval.

Hence, the possibility of considering non-commutative conjunctors provides more flexibility and increases the number of applications.

This is one of the properties that the multi-adjoint concept lattice framework offers. Another important feature is that different preferences among the set of attributes or/and objects can be considered.

Moreover, this framework has been extended following different lines and has been applied to define general extensions of fuzzy rough sets theory, to solve fuzzy relation equations, etc.
\end{abstract}

\section{References}

1. P. Julian, G. Moreno, and J. Penabad. On fuzzy unfolding: A multi-adjoint approach. Fuzzy Sets and Systems, 154(1):16-33, 2005.

2. J. Medina, M. Ojeda-Aciego, and J. Ruiz-Calviño. Formal concept analysis via multi-adjoint concept lattices. Fuzzy Sets and Systems, 160(2):130-144, 2009.

3. J. Medina, M. Ojeda-Aciego, and J. Ruiz-Calviño. On multi-adjoint concept lattices: definition and representation theorem. Lecture Notes in Artificial Intelligence, 4390:197-209, 2007.

4. J. Medina, M. Ojeda-Aciego, and P. Vojtáš. Similarity-based unification: a multiadjoint approach. Fuzzy Sets and Systems, 146:43-62, 2004.

\footnotetext{
* Partially supported by Spanish Ministry of Science and FEDER funds through project TIN09-14562-C05-03 and Junta de Andaluca project P09-FQM-5233.
} 